\title{
Mindset Trek
}

\section{Elearning for Developing Personal Innovativeness_-A Case Study}

https://doi.org/10.3991/ijac.v14i2.25845

\author{
Dennis Stauffer ${ }^{(凶)}$ \\ Innovator Mindset, Minneapolis, Minnesota, United States \\ dstauffer@innovatormindset.com
}

\begin{abstract}
Throughout the world today, the capacity to innovate is no longer an option. It's a necessity for any organization that hopes to survive and find success. However, innovation is not just an organizational capability. A crucial piece is the personal capabilities of those who are called on to enable that innovation. That's arguably everyone. Mindset Trek elearning is designed to develop the mindset needed to be an effective innovator, in a wide range of settings: settings that include universities and startup incubators and accelerators, as well as corporate, $\mathrm{R} \& \mathrm{D}$, government and non-profit. By focusing on the underlying mindset that is common to all types of innovation, this approach transcends those boundaries.
\end{abstract}

Keywords-innovation training, mindset training, elearning, adaptive elearning, assessing personal innovativeness

\section{The challenge}

Recent research has found that the personal capacity to innovate - innovativeness - is determined less by knowledge and skills than by mindset. That is the largely unconscious assumptions and beliefs we all hold. Thus, developing personal innovativeness poses unique challenges that are different from those faced by other types of elearning curriculum.

Traditional approaches to developing knowledge and skills involve transmitting the needed knowledge and practicing the requisite skills. Shifting mindset involves developing self-awareness, revealing options students may not realize they have, and motivating students to make choices that drive innovation. Therefore, it requires a specifically sequenced intervention that:

1) Provides sufficiently motivating reasons for students to shift their mindset

2) Explains alternative mindsets that strengthen or undermine innovation capabilities.

3) Delivers personalized diagnostic feedback on the mindset a student currently has.

4) Gives specific guidance on what shifts a student can make to enhance innovativeness.

5) Provides strategies for internalizing an Innovator Mindset.

All this must be delivered in a way that recognizes it is the student who must decide whether to make the recommended shifts. 


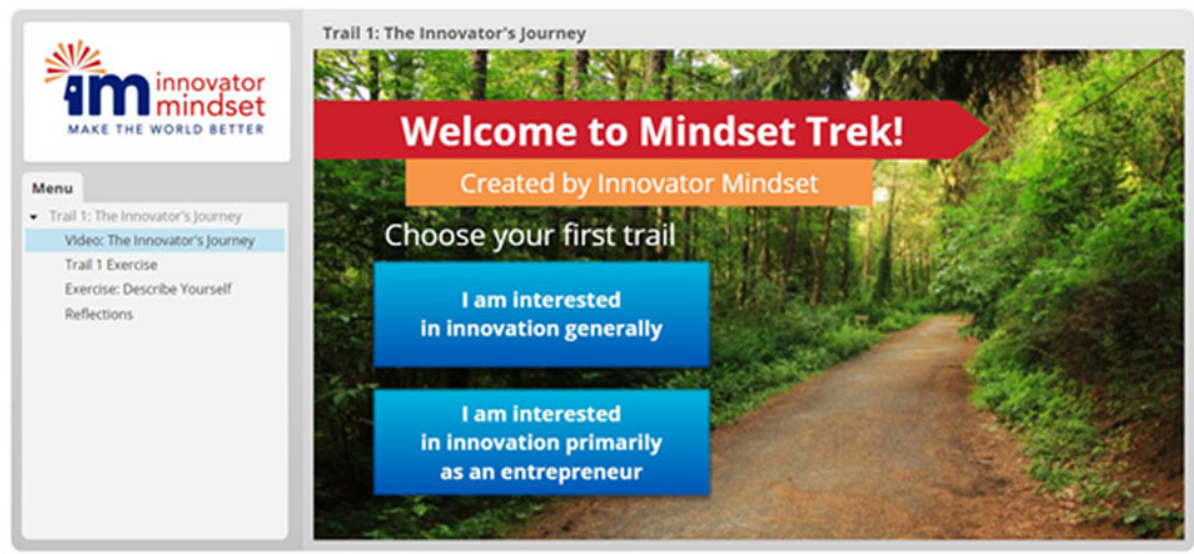

\section{$2 \quad$ Mindset-focused elearning}

Mindset Trek is an elearning program that takes up that challenge. It begins by making the case for personal innovativeness and explains the many benefits it provides. Students then take the rigorously validated Innovator Mindset ${ }^{\mathbb{}}$ self-assessment that measures their mindset across twelve dimensions. They are then taken through a series of lessons that explain what an innovator mindset is and what it requires. Each student then receives personalized feedback based on their personal assessment results and adaptive guidance on what shifts they can make to become more innovative.

Once that feedback and guidance has been provided, students are given content on how to internalize an Innovator Mindset and begin practicing the habits of highly effective innovators.

The overall learning objectives are to:

- Increase students' self-awareness of their mindset.

- Reveal innovation-relevant choices students may not have recognized.

- Help students choose wise and personally rewarding options.

- Prompt students to practice applying that mindset.

To accomplish these things, the elearning content must be compelling, personally relevant and immediately actionable.

The unique approach taken by Innovator Mindset and Mindset Trek complements a wide range of innovation tools and strategies, including creative problem solving, design thinking, lean startup and the scientific method. So, this elearning can be paired with - or ideally used to introduce and enhance-those approaches. This makes it possible for Mindset Trek to be implemented as a stand-alone offering, or as part of blended learning. Instructors have access to a comprehensive collection of exercises and assignments that reinforce and deepen the elearning content. 


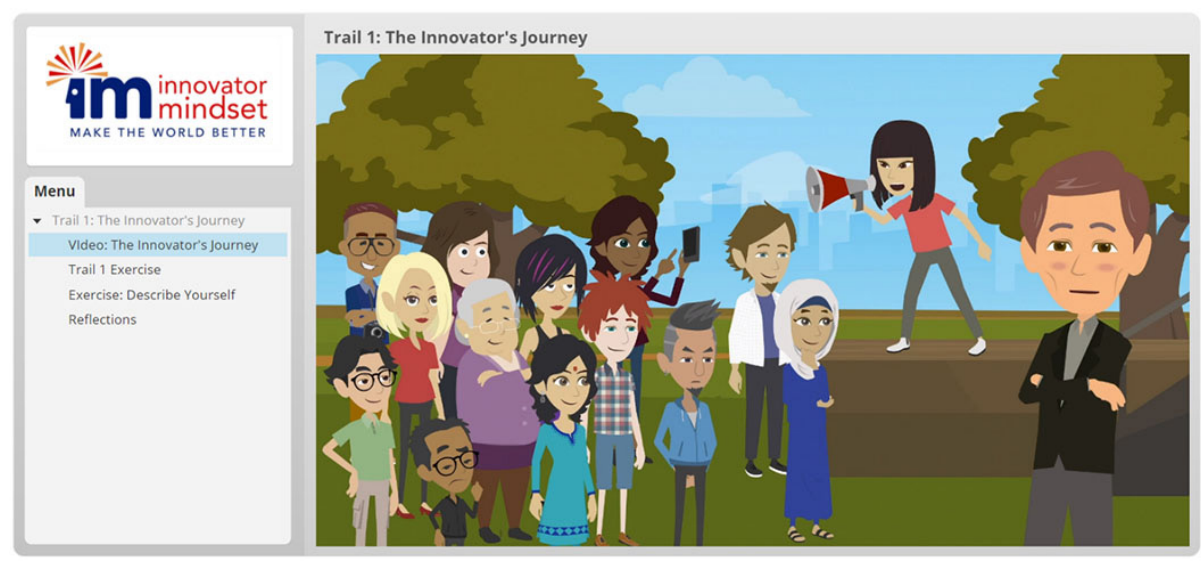

\section{Delivery}

Mindset Trek elearning content is delivered through a combination of illustrative videos, animations, on-camera expert guidance, and interactive quizzes and exercises that gauge comprehension. Students are required to demonstrate mastery of each Trail's key concepts before moving to the next Trail.

The Innovator Mindset assessment is integrated into the elearning through a direct link to the online survey and an interactive elearning dashboard that provides personal results and feedback. That adaptive content is delivered strategically at appropriate times in the learning sequence.

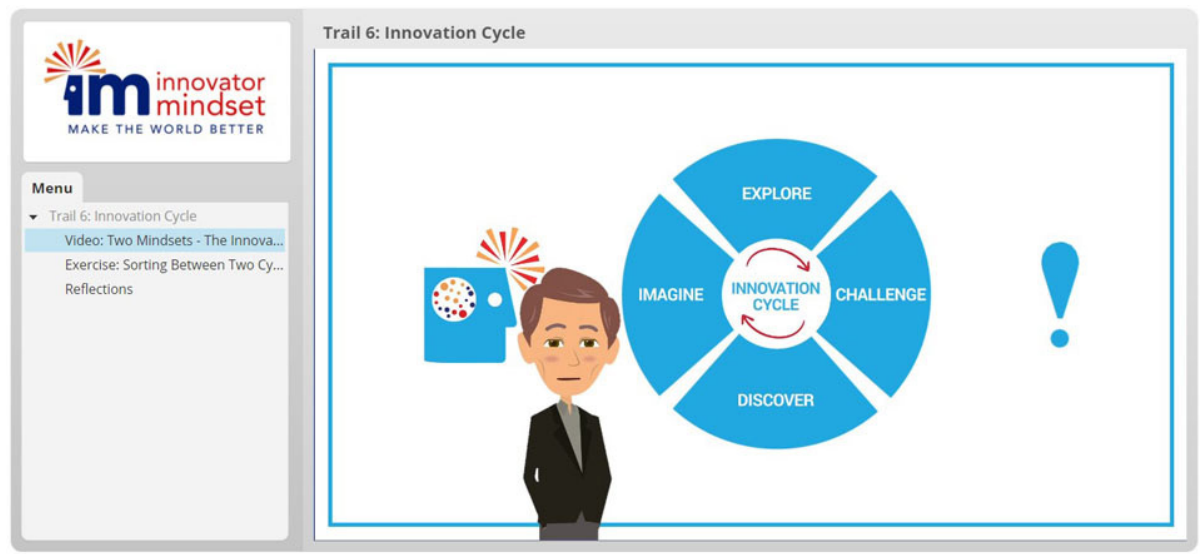

\section{$4 \quad$ Learning sequence}

Each trail in Mindset Trek begins with a short video, followed by a brief exercise or quiz to confirm understanding of the material covered. Students are urged to capture 
their questions, ideas and insights, and reflect on that material. They're free to continue Mindset Trek at their own pace, or as directed by an instructor, coach or mentor. The length of each Trail varies, but they typically take about 20 minutes to complete.

Trail 1 lets students choose to take this journey as an entrepreneur, or as an innovation generalist. The core content is the same, but the application may be different, so two different introductions are provided.

Trains 1-3 explain what this journey will look like and its benefits. After completing Trail 3, students are prompted to take the Innovator Mindset assessment. This takes about 15 minutes to complete online. Once they have finished the assessment, they resume their journey.

Trails 4-7 explain what an Innovator Mindset is and introduce key concepts needed to understand and interpret the Innovator Mindset assessment results.

In Trail 8, students gain access to a personal dashboard that provides detailed feedback based on the Innovator Mindset assessment. They can go back to this dashboard to review their results at any time.

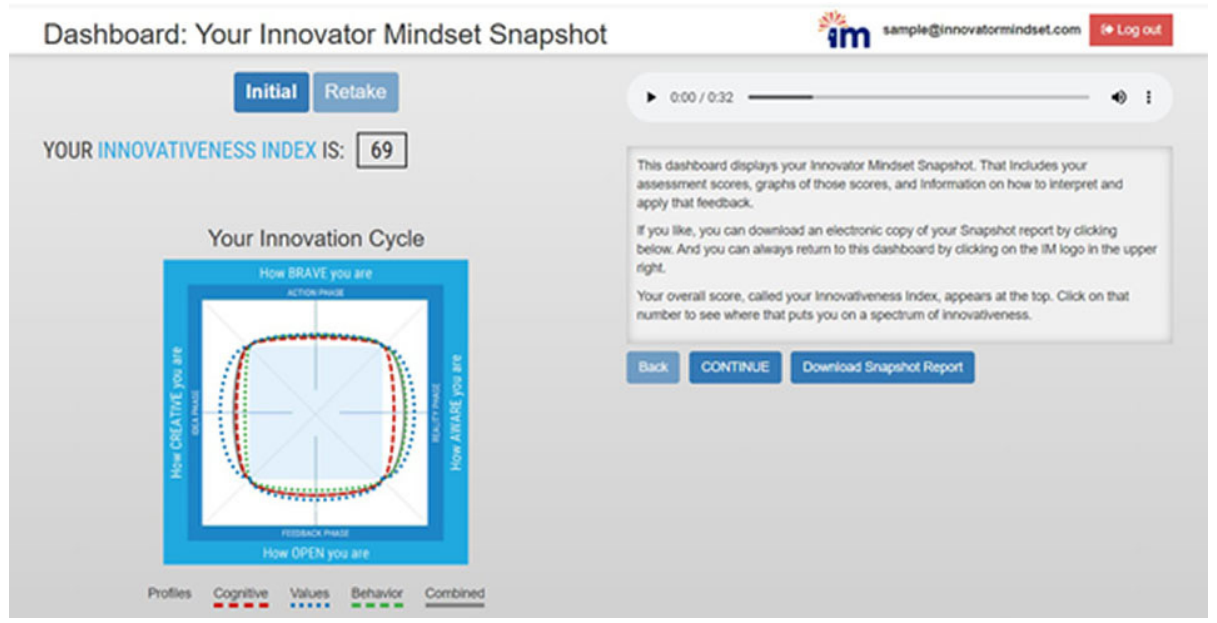

Trail 9 introduces students to a series of four Paths they can choose, depending on where they are as an innovator and the challenges they're pursuing. Once they select a place to start, they're guided through the rest of the Innovation Cycle.

Trail 10 gives guidance for each of those Paths and how to navigate that Phase of the Innovation Cycle. Students also learn the Habits of an Innovator for each Phase. They're prompted to return to their Innovator Mindset dashboard to get personalized feedback within each Phase, and they are urged to begin practicing those habits.

Trail 11 explains how to navigate the Innovation Cycle as a whole, while keeping things in balance, and directs students back to their IM dashboard for personalized feedback on how well they may be doing that.

Trail 12 completes the journey by giving students an opportunity to retake the Innovator Mindset assessment. Their results are immediately available in their dashboard, so they can compare them to their previous scores to gauge their progress in developing an Innovator Mindset. 
Mindst Trek and the Innovator Mindset assessment are available online on demand and can be scaled to any number of users. This content has been developed prioritizing diversity and accessibility.

For more information:

Innovator Mindset Website: https://innovatormindset.com

Mindset Trek Elearning: https://innovatormindset.com/mindset-trek-elearning/

Research: https://innovatormindset.com/research/

\section{$5 \quad$ Author}

Dennis Stauffer is an independent researcher and founder of Innovator Mindset LLC. He led the team that produced Mindset Trek, based in part on research done in cooperation with the Ewing Marion Kauffman Foundation and innovativeness work done under a grant from the U.S. National Science Foundation.

Article submitted 2021-07-29. Resubmitted 2021-09-21. Final acceptance 2021-09-23. Final version published as submitted by the authors. 